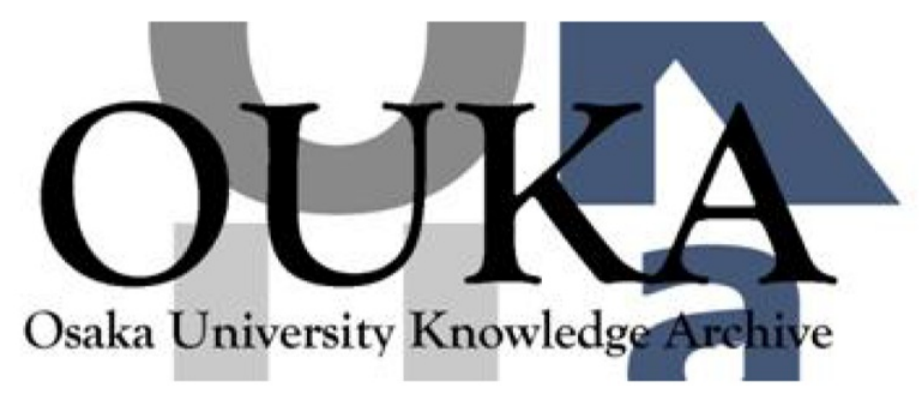

\begin{tabular}{|c|l|}
\hline Title & The Valve Action of Intensity Receptor \\
\hline Author(s) & Tsukamoto, Yoshihiko \\
\hline Citation & \\
\hline Issue Date & \\
\hline oaire:version & VoR \\
\hline URL & https://hdl. handle. net/11094/31321 \\
\hline rights & \\
\hline Note & \\
\hline
\end{tabular}

Osaka University Knowledge Archive : OUKA

https://ir. Library. osaka-u. ac. jp/

Osaka University 


\title{
The Valve Action of Intensity Receptor
}

\author{
Yoshiniko Tsukamoto \\ Department of Biology, Faculty of Science, \\ Osaka University, Toyonaka, Osaka, Japan \\ (Received 4 April 1972, and in revised form 21 May 1973)

\begin{abstract}
The valve action of intensity receptor has the following properties which are essential to an encoder. (1) The response amplitude corresponds to the stimulus intensity in a one-to-one manner. (2) The dynamic ranges of the stimulus intensity and the response amplitude are respectively set and able to move in parallel with their axes. (3) The probability density distribution of the stimulus intensity may be transformed into that of the response amplitude by a stimulus-response relation. Concerning stimulus-response relations, three empirical formulae, i.e. logarithmic, power and tanh log functions, are well known. These formulae can be arranged for the forms to express the above three properties. Then, power and tanh $\log$ functions are converted to a logarithmic function as the exponents of them approach zero. From a viewpoint of information theory, the stimulus-response transformation may play a role to improve the probabilistic nature of stimuli in order to make an efficient code for a given channel of sensory fibre.
\end{abstract}

\section{Introduction}

The valve action of intensity receptor is the stimulus of different forms (energies or chemical substances) controlling the flow of ionic current through a cell membrane. The stimulus intensity is transformed into the amplitude of slow potential with non-linear relationships. It is as if grid potential would control the flow of plate current in a vacuum tube amplifier with grid characteristics. Slow potential evokes propagative impulse discharges at the root of axon (Eyzaguirre \& Kuffler, 1955). The potential amplitude is transformed into the impulse frequency proportionally (Katz, 1950; Fuortes, 1958, 1970). Thus, as we can see in a typical model of communication system (Fano, 1963), the encoding is performed by two steps in the intensity receptor. First there is a source encoder dependent on the characteristics of an information source, which transforms the stimulus intensity $i$ into the potential amplitude $v$ non-linearly. The other is a channel encoder dependent on the characteristics of a communication 
channel, which transforms the potential amplitude into the impulse frequency $f$ linearly.

So far, concerning the relationships between the stimulus intensity and the potential amplitude (or the impulse frequency), three empirical formulae are well known. The first is a logarithmic function called Weber-Fechner's law,

$$
h_{1}(i)=a \log i+b
$$

which has been recorded by a number of electrophysiological investigations of various sensory systems at least in the intermediate range of stimulus intensities (Matthews, 1931; Hartline \& Graham, 1932; Galambos \& Davis, 1943). The second is a power function introduced by Stevens (1961) at first in psychophysics,

$$
h_{2}(i)=c i^{n}+d .
$$

Werner \& Mountcastle (1965) studied the neural activity of mechanoreceptive first-order sensory fibers, and showed that the power function holds with a high degree of statistical validity. The third is a tanh (hyperbolic tangent) $\log$ function,

$$
h_{3}(i)=\frac{i^{n}}{i^{n}+\alpha^{n}}=\frac{1}{2}+\frac{1}{2} \tanh \left(\frac{n}{2} \log \frac{i}{\alpha}\right)
$$

which was reported recently (Boynton \& Whitten, 1970; Ernst \& Kemp, 1972). This function in general shows sigmoidal shapes. As a special case that the exponent $n$ is equal to one, it reduces to the same form as the adsorption isotherm of Langmuir which is recorded in some sense organs (Beidler, 1954; Baylor \& Fuortes, 1970).

We assume that the stimulus intensity and the impulse frequency are several sets of random variables and the probability density distributions of them are well-defined. It is an important feature that the non-linear one-to-one transformation from the stimulus intensity to the impulse frequency also accompanies the conversion of the probability density distribution of the former into that of the latter. The change of the probability density distributions is one of the essential meanings of the stimulusresponse relation from a viewpoint of information theory. Stein (1967) calculated the capacity of nerve cells to transmit information using a frequency code. The mutual information transmitted by a channel is the response uncertainty minus the conditional uncertainty. The conditional uncertainty depends on the noise characteristics of a given channel. The response uncertainty is a function of the probability density function (p.d.f.) of the mean impulse frequency which depends on the stimulus intensity via the stimulus-response relation. The channel capacity is obtained when the p.d.f. of the mean impulse frequency is optimal so as to give the maximum 
of the mutual information. It is considered that the stimulus-response relation may play a role to improve the probabilistic nature of stimuli in order to make an efficient code for a given channel of sensory fibre.

\section{Properties of Valve Action}

(1) The source of stimulus energy and the source of response energy are independent of each other.

(a) Various forms of stimulus energies (chemical substances are considered a form of energy, i.e. chemical potential) in the outer environment are convertible into a common form of response energies (i.e. receptor potential) to perform the internal transmission of information. In fact, there are many sense organs of different kinds, each response amplitude of which may be controlled by the stimulus of its modality.

(b) In general the response amplitude is controlled by the relatively small amount of the stimulus energy. The ratio of the amount of energy involved in response to the amount of energy involved in stimulus can vary with certain freedom. The variability of this ratio is a necessary condition of the adjustment of dynamic ranges (i.e. gain control).

(2) The stimulus intensity prescribes the response amplitude.

(a) The response amplitude corresponds to the stimulus intensity in one-to-one manner. One-to-one correspondence between two variables is a necessary condition for uniquely decipherable encoding in receptor.

(b) The dynamic ranges of the stimulus intensity and the response amplitude are respectively set and able to move in parallel with their axes. The adjustment of dynamic ranges is the adaptation of receptor to the occasional changes of a stimulus condition in outer environment.

(c) The probability density distribution of the stimulus intensity may be transformed into that of the response amplitude by a stimulusresponse relation. A non-linear transformation between them is necessary to make an efficient code for a communication channel of sensory fiber. The amount of information transmitted is defined by an entropy function of the p.d.f. of the response variable, presuming the noise characteristics of channel.

\section{Formalization of Stimulus-Response Relations}

The response amplitude is a function of the stimulus intensity. Three empirical formulae (i.e. logarithmic, power and tanh log functions) are onevalue, continuous and strictly increasing functions. These represent the one- 
to-one correspondence between the stimulus and the response variables and also the non-linearity in that the first differentials are not constant. The usual expressions of empirical formulae do not represent the dynamic ranges of the stimulus intensity and the response amplitude explicitly. The dynamic range defines a set of random variables, on which probabilities are well defined. Namely, the probability distributions on the sets of the stimulus intensity and the response amplitude which are the random variables are not defined until both dynamic ranges are set.

An empirical formula $h(i)$ represents one-to-one and non-linear transformations from the stimulus intensity into the potential amplitude [Fig. 1(a)].

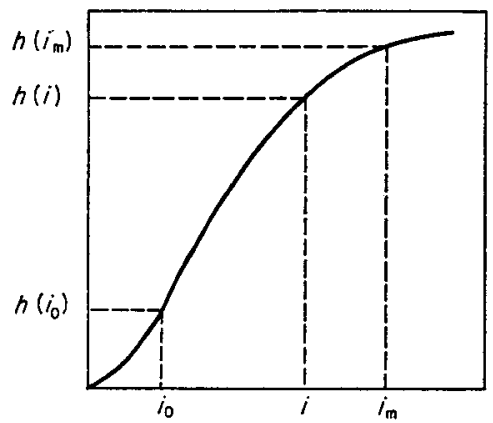

(a)

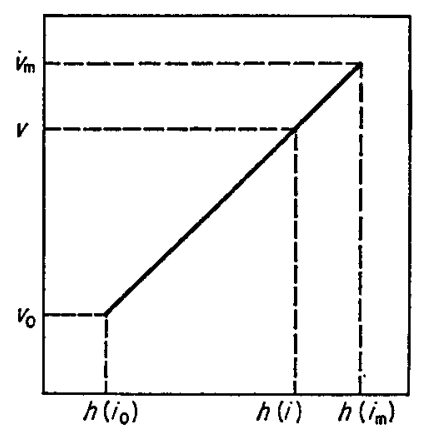

(b)

Fig. 1. (a) One-to-one and non-linear transformation from the stimulus intensity into the potential amplitude. (b) The adjustment of dynamic ranges of the stimulus intensity and the potential amplitude.

The dynamic ranges of the stimulus intensity and the potential amplitude can move in parallel with their axes respectively (Naka \& Kishida, 1966). Both are inter-related proportionally [Fig. 1(b)]. Then,

$$
v=A h(i)+B \text {. }
$$

The minimum $i_{0}$ and the maximum $i_{\mathrm{m}}$ of the stimulus intensity correspond to the minimum $v_{0}$ and the maximum $v_{\mathrm{m}}$ of the potential amplitude,

$$
\begin{aligned}
& v_{0}=A h\left(i_{0}\right)+B \\
& v_{\mathrm{m}}=A h\left(i_{\mathrm{m}}\right)+B .
\end{aligned}
$$

$A$ and $B$ satisfying the simultaneous equations of (5) and (6) are derived as

$$
\begin{aligned}
A & =\frac{v_{\mathrm{m}}-v_{0}}{h\left(i_{\mathrm{m}}\right)-h\left(i_{0}\right)} \\
B & =\frac{v_{0} h\left(i_{\mathrm{m}}\right)-v_{\mathrm{m}} h\left(i_{0}\right)}{h\left(i_{\mathrm{m}}\right)-h\left(i_{0}\right)} .
\end{aligned}
$$


Substituting equations (7) and (8) into equation (4), the following proportional expression is obtained,

$$
\frac{v-v_{0}}{v_{\mathrm{m}}-v_{0}}=\frac{h(i)-h\left(i_{0}\right)}{h\left(i_{\mathrm{m}}\right)-h\left(i_{0}\right)}
$$

Hence, an intensity-amplitude relation is

$$
v=g(i)=\left(v_{\mathrm{m}}-v_{0}\right) \frac{h(i)-h\left(i_{0}\right)}{h\left(i_{\mathrm{m}}\right)-h\left(i_{0}\right)}+v_{0} .
$$

In the case of logarithmic function, substituting equation (1) into equation (10) gives

$$
g_{1}(i)=\left(v_{\mathrm{m}}-v_{0}\right) \frac{\log i-\log i_{0}}{\log i_{\mathrm{m}}-\log i_{0}}+v_{0} .
$$

In the case of power function, substituting equation (2) into equation (10) gives

$$
g_{2}(i)=\left(v_{\mathrm{m}}-v_{0}\right) \frac{i^{n}-i_{0}^{n}}{i_{\mathrm{m}}^{n}-i_{0}^{n}}+v_{0} .
$$

In the case of tanh $\log$ function, some remarks are necessary for constant $\alpha$. Regarding the empirical formula, $h(i)=1 / 2$ is given for $i=\alpha . \alpha$ has been introduced at such a value that the response amplitude might become equal to half of the maximum when the stimulus intensity equals $\alpha$. Then, in order that $v=1 / 2$ might be given for $i=\alpha$,

$$
\alpha=\left(i_{\mathrm{m}} i_{0}\right)^{1 / 2}
$$

is a necessary condition. Substituting equations (3) and (13) into equation (10) gives

$$
\begin{aligned}
g_{3}(i) & =\left(v_{\mathrm{m}}-v_{0}\right) \frac{\frac{i^{n}}{i^{n}+\left(i_{\mathrm{m}} i_{0}\right)^{n / 2}}-\frac{i_{0}^{n}}{i_{\mathrm{m}}^{n}}-\frac{i_{0}^{n}+\left(i_{\mathrm{m}} i_{0}\right)^{n / 2}}{i_{0}^{n}}}{i_{\mathrm{m}}^{n}+\left(i_{\mathrm{m}} i_{0}\right)^{n / 2}}-v_{0} \\
& =\left(v_{\mathrm{m}}-v_{0}\right)\left(\frac{\tanh \left\{\frac{n}{2} \log \frac{i}{\left(i_{\mathrm{m}} i_{0}\right)^{n / 2}}\right.}{2 \tanh \left(\frac{n}{4} \log \frac{i_{\mathrm{m}}}{i_{0}}\right)}+\frac{1}{2}\right)+v_{0} .
\end{aligned}
$$

Consider the following part of equation (12),

$$
\frac{i^{n}-i_{0}^{n}}{i_{\mathrm{m}}^{n}-i_{0}^{n}}
$$


which is a function of the exponent $n$. This function is satisfied with the propositions that are necessary for the application of L'Hospital's theorem.

(1) Differentiations of numerator and denominator are possible.

$$
\begin{aligned}
& \frac{\mathrm{d}}{\mathrm{d} n}\left(i^{n}-i_{0}^{n}\right)=i^{n} \log i-i_{0}^{n} \log i_{0} \\
& \frac{\mathrm{d}}{\mathrm{d} n}\left(i_{\mathrm{m}}^{n}-i_{0}^{n}\right)=i_{\mathrm{m}}^{n} \log i_{\mathrm{m}}-i_{0}^{n} \log i_{0} .
\end{aligned}
$$

(2) A differential of denominator is not equal to zero.

$$
\frac{\mathrm{d}}{\mathrm{d} n}\left(i_{\mathrm{m}}^{n}-i_{0}^{n}\right) \neq 0 \quad\left(\because i_{\mathrm{m}} \neq i_{0}\right)
$$

(3) A fractional expression (15) takes an indeterminate form $0 / 0$ for $n=0$.

$$
i^{0}-i_{0}^{0}=i_{\mathrm{m}}^{0}-i_{0}^{0}=1-1=0 .
$$

(4) A limiting value of the fractional expression of differentials as the exponent $n$ approaches zero exists.

$$
\begin{aligned}
\lim _{n \rightarrow 0} \frac{\frac{\mathrm{d}}{\mathrm{d} n}\left(i^{n}-i_{0}^{n}\right)}{\frac{\mathrm{d}}{\mathrm{d} n}\left(i_{\mathrm{m}}^{n}-i_{0}^{n}\right)} & =\lim _{n \rightarrow 0} \frac{i^{n} \log i-i_{0}^{n} \log i_{0}}{i_{\mathrm{m}}^{n} \log i_{\mathrm{m}}-i_{0}^{n} \log i_{0}} \\
& =\frac{\log i-\log i_{0}}{\log i_{\mathrm{m}}-\log i_{0}}
\end{aligned}
$$

Therefore, taking the limit of formula (15) as the exponent $n$ approaches zero yields

$$
\lim _{n \rightarrow 0} \frac{i^{n}-i_{0}^{n}}{i_{\mathrm{m}}^{n}-i_{0}^{n}}=\frac{\log i-\log i_{0}}{\log i_{\mathrm{m}}-\log i_{0}} .
$$

This is equivalent to the part of equation (11). Similarly, taking the limit of the part of equation (14a) as the exponent $n$ approaches zero yields

$$
\lim _{n \rightarrow 0} \frac{\frac{i^{n}}{i^{n}+\left(i_{\mathrm{m}} i_{0}\right)^{n / 2}}-\frac{i_{o}^{n}}{i_{0}^{n}+\left(i_{\mathrm{m}} i_{0}\right)^{n / 2}}}{\frac{i_{\mathrm{m}}^{n}}{i_{\mathrm{m}}^{n}+\left(i_{\mathrm{m}} i_{0}\right)^{n / 2}}-\frac{i_{0}^{n}}{i_{0}^{n}+\left(i_{\mathrm{m}} i_{0}\right)^{n / 2}}}=\frac{\log i-\log i_{0}}{\log i_{\mathrm{m}}-\log i_{0}} .
$$

This is equivalent to the part of equation (11).

Thus in the limit as the exponent $n$ approaches zero, equations (12) and (14) change into equation (11). We show the changes of the power functions and the tanh $\log$ functions into the logarithmic function for several values of the exponent in graphic form. In the following, dynamic ranges are set 


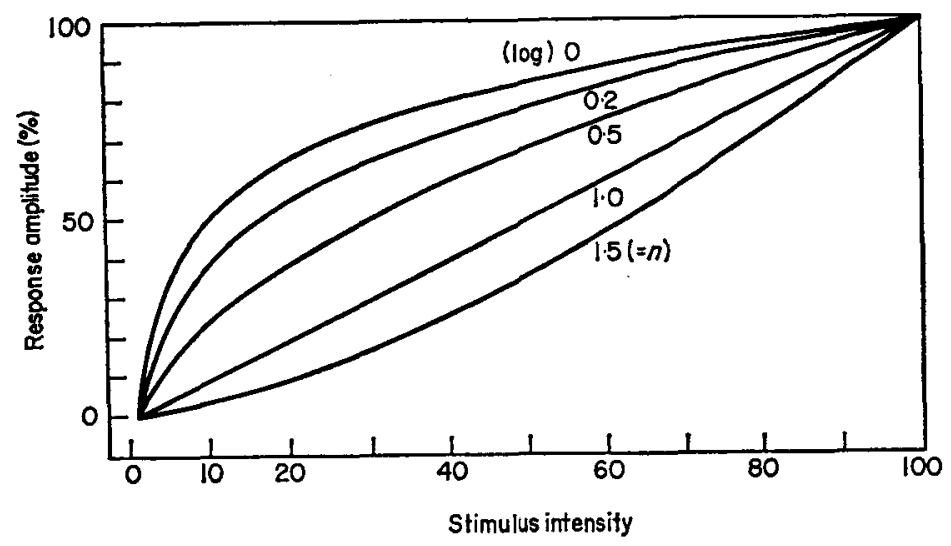

FIG. 2. The intensity-amplitude relations of power functions. For $n=1.5,1.0,0.5$ and $\mathbf{0 \cdot 2}$

For $n=0(\log )$

$$
v=\frac{100}{100^{n}-1}\left(i^{n}-1\right)
$$

$$
v=\frac{100}{\log 100} \log i
$$

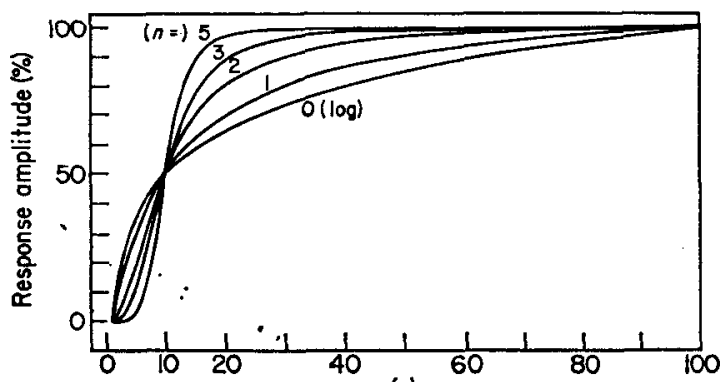

(a)

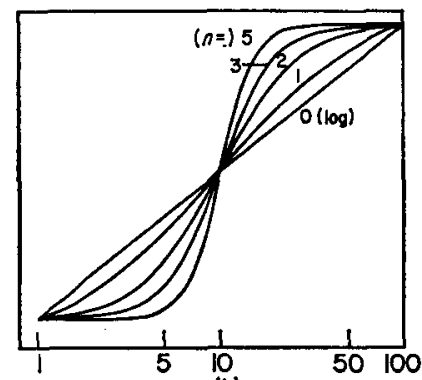

(b)

Frg. 3. (a) The intensity-amplitude relations of $\tanh \log$ functions. For $n=5,3,2$ and 1

For $n=0(\log )$

$$
\begin{aligned}
v & =100 \frac{10^{n}+1}{10^{n}-1}\left(\frac{\mathrm{i}^{n}}{\mathrm{i}^{n}+10^{n}}-\frac{1}{1+10^{n}}\right) \\
& =100 \frac{\tanh \left(\frac{n}{2} \log \frac{i}{10}\right)}{2 \tanh \left(\frac{n}{4} \log 100\right)}+\frac{1}{2} .
\end{aligned}
$$

$$
v=\frac{100}{\log 100} \log i
$$

(b) Fig. 3 (a) redrawn against the logarithmic scale of the stimulus intensity. 
as $i_{0}=1, i_{\mathrm{m}}=100, v_{0}=0$ and $v_{\mathrm{m}}=100$. Intensity-amplitude curves of power functions are illustrated in Fig. 2 by using equations (11) and (12) for $n=1.5,1.0,0.5,0.2$ and 0 (corresponding to the logarithm). Intensityamplitude curves of tanh $\log$ functions are illustrated in Fig. 3(a) and also 3 (b) by using equations (11) and $(14 a, b)$ for $n=5,3,2,1$ and 0 (corresponding to the logarithm). The abscissa of the stimulus intensity is plotted out by the uniform scales in Fig. 3(a), and again by the logarithmic scale in Fig. 3(b). Curves shown in Fig. 3(b) have a point of symmetry.

\section{Relationship of Probability Distributions}

We suppose that a series of intensities of the stimuli coming into a single intensity receptor is composed of statistically independent random variables which constitute a stationary stochastic process. Let the stimulus intensity $I=\left(i \mid i_{0} \leqslant i \leqslant i_{\mathrm{m}}\right)$ be a set of one-dimensional, continuous random variables, then the response amplitude $V=\left(v \mid v_{0} \leqslant v \leqslant v_{\mathrm{m}}\right)$ which is dependent on the stimulus intensity by a one-to-one representation $V=g(I)$ becomes a set of one-dimensional, continuous random variables too.

Let $\psi(i)$ be a function showing a probability density distribution of the stimulus intensity, and $\Psi(i)$ be a primitive function of $\psi(i)$. A p.d.f. of the stimulus intensity is

$$
q(i)=\frac{\psi(i)}{\Psi\left(i_{\mathrm{m}}\right)-\Psi\left(i_{0}\right)} .
$$

And a distribution function corresponding to $q(i)$ is

$$
Q(i)=\frac{\Psi(i)-\Psi\left(i_{0}\right)}{\Psi\left(i_{\mathrm{m}}\right)-\Psi\left(i_{0}\right)} .
$$

Let $\phi(v)$ be a function showing a probability density distribution of the response amplitude, and $\Phi(v)$ be a primitive function of $\phi(v)$. A p.d.f. of the response amplitude is

$$
p(v)=\frac{\phi(v)}{\Phi\left(v_{\mathrm{m}}\right)-\Phi\left(v_{0}\right)} .
$$

A distribution function corresponding to $p(v)$ is

$$
P(v)=\frac{\Phi(v)-\Phi\left(v_{0}\right)}{\Phi\left(v_{\mathrm{m}}\right)-\Phi\left(v_{0}\right)} .
$$

The intensity-amplitude transformation $v=g(i)$ is one-value, continuous and strictly monotonic function. Therefore, a relation between the p.d.f.'s 
of the stimulus intensity and the response amplitude is

$$
q(i)=p\{g(i)\} \frac{\mathrm{d} g(i)}{\mathrm{d} i} .
$$

Namely,

$$
\frac{\psi(i)}{\Psi\left(i_{\mathrm{m}}\right)-\Psi\left(i_{0}\right)}=\frac{\phi\{g(i)\}}{\Phi\left\{g\left(i_{\mathrm{m}}\right)\right\}-\Phi\left\{g\left(i_{0}\right)\right\}} \frac{\mathrm{d} g(i)}{\mathrm{d} i} .
$$

A relation between the distribution functions of them is

Namely,

$$
P(v)=Q(i)=P\{g(i)\}
$$

$$
\frac{\Phi(v)-\Phi\left(v_{0}\right)}{\Phi\left(v_{\mathrm{m}}\right)-\Phi\left(v_{0}\right)}=\frac{\Psi(i)-\Psi\left(i_{0}\right)}{\Psi\left(i_{\mathrm{m}}\right)-\Psi\left(i_{0}\right)}=\frac{\Phi\{g(i)\}-\Phi\left\{g\left(i_{0}\right)\right\}}{\Phi\left\{g\left(i_{\mathrm{m}}\right)\right\}-\Phi\left\{g\left(i_{0}\right)\right\}}
$$

Hence, if any two of $P(v), Q(i)$ and $v=g(i)$ are settled, another one can be determined by relations (24).

\section{Increment Threshold}

When an increment $\Delta v$ of the response amplitude corresponds to an increment $\Delta i$ of the stimulus intensity, taking the first approximation of Taylor's series,

$$
\Delta v=g(i+\Delta i)-g(i) \sim \Delta i g^{\prime}(i) .
$$

Let $\Delta v$ be an increment threshold of the potential amplitude, which is determined by the variability of neural activity. Then,

$$
\Delta i=\Delta v \frac{1}{g^{\prime}(i)}
$$

is an increment threshold of the stimulus intensity. Weber's ratio is expressed as

$$
\frac{\Delta i}{i}=\frac{\Delta v}{i g^{\prime}(i)}
$$

\section{Channel Capacity}

Stein (1967) derived approximate equations for the amount of information a nerve cell can transmit about a stimulus of a given duration using a frequency code. The characteristics of a stimulus in outer environment and a stimulus-response relation in sensory receptor is beyond the scope of his study. We shall form a connection between the above-stated results and some general results derived by him. 
Impulse discharges inherently have variability. Stein (1967) made a summary of experimental findings in which the standard deviation $\sigma$ of the interspike interval distribution is a power function of the mean interspike interval $\mu$. Then,

$$
\sigma=b \mu^{r}
$$

where $b$ and $r$ are constants and generally $r \geqslant 1$. He introduced an important result based on the central limit theorem of mathematics (Cox \& Miller, 1965). It can be stated as follows: if the intervals between successive nerve impulses are independent, random variables (renewal process) whose probability distribution has mean $\mu$ and variance $\sigma^{2}$, the distribution of the number of nerve impulses is asymptotically normal as $t \rightarrow \infty$, with $x \sim t / \mu$ and variance

$$
s^{2} \sim \sigma^{2} t / \mu^{3} .
$$

According to his model, the stimulus is defined in terms of the mean number of impulses during a given period it generates. The optimum p.d.f. $\omega(x)$ of the mean number of impulses necessary to attain the channel capacity of a nerve fibre is given by

$$
\omega(x)=D / s(x)
$$

where $D$ is a constant such that

$$
D^{-1}=\int_{x_{\min }}^{x_{\max }} \frac{\mathrm{d} x}{s(x)}
$$

Equation (31) states that the p.d.f. for the occurrence of particular stimuli (i.e. mean number of impulses) must vary inversely as the standard deviation in number of impulses. We assume that the potential amplitude may be transformed into the mean impulse frequency $f$ proportionally at channel encoding,

$$
\frac{v-v_{0}}{v_{\mathrm{m}}-v_{0}}=\frac{f-f_{0}}{f_{\mathrm{m}}-f_{0}} .
$$

Then the impulse frequency corresponds to the stimulus intensity via the transformation of $v=g(i)$. Assuming that each duration $t$ of successive and individual stimuli is sufficiently large and constant, the mean impulse frequency $f$ can be adopted as variable instead of the mean number of impulses $x$ which is used in his paper. The averages of the interspike interval and the impulse frequency during a given period, have a reciprocal relation $\mu=1 / f$. Then, the mean number of impulses is also expressed as

$$
x=f t \text {. }
$$

Substituting equations (29) and (34) into equation (30) yields

$$
s \sim b t^{1 / 2} f^{3 / 2-r} \text {. }
$$


A calculation of equations (31) and (32) by using equations (34) and (35) yields

$$
\omega(f t)=\frac{1}{t} \frac{k f^{k-1}}{f_{\mathrm{m}}^{k}-f_{0}^{k}}
$$

where $k=r-0 \cdot 5$. Changing the variable from $x$ into $f$, the optimum p.d.f. $p(f)$ of the mean impulse frequency is given by

$$
p(f)=\omega(x) \frac{\mathrm{d} x}{\mathrm{~d} f}=\omega(f t) t .
$$

Therefore, the optimum p.d.f. of the mean frequency is

$$
p(f)=\frac{k f^{k-1}}{f_{\mathrm{m}}^{k}-f_{0}^{k}}
$$

In this case, the channel capacity of the nerve fibre which is characterized by equation (29), is approximated as

$$
C \sim \log \left[\sqrt{\frac{t}{2 \pi e}} \frac{f_{\mathrm{m}}^{k}-f_{0}^{k}}{b k}\right]
$$

which follows in one step from equation (2.12) of Stein (1967).

Since the potential amplitude is proportional to the mean impulse frequency, the optimum p.d.f. of the potential amplitude is

$$
p(v)=\frac{k v^{k-1}}{v_{\mathrm{m}}^{k}-v_{0}^{k}} \text {. }
$$

The distribution function corresponding to $p(v)$ is

$$
P(v)=\frac{v^{k}-v_{0}^{k}}{v_{\mathrm{m}}^{k}-v_{0}^{k}}
$$

When a sensory nerve characterized by the stimulus-response relation $v=g(i)$ and the coefficient $k$ acts at the full capacity of itself, the distribution functions of the stimulus intensity and the response amplitude is related as

by using equation (25).

$$
\frac{v^{k}-v_{0}^{k}}{v_{\mathrm{m}}^{k}-v_{0}^{k}}=\frac{g(i)^{k}-g\left(i_{0}\right)^{k}}{g\left(i_{\mathrm{m}}\right)^{k}-g\left(i_{0}\right)^{k}}
$$

\section{Remarks and Discussion}

Consider a special case of sensory nerve fibre whose noise characteristics are given by $k=1$ (or $r=1 \cdot 5$, i.e. the standard deviation $s$ is constant). We assume that the increment threshold of the potential amplitude $\Delta v$ may be determined by the corresponding increment threshold of the impulse 
frequency $\Delta f$, then proportional to the standard deviation $s$. If $s$ is constant, so $\Delta v$ is too.

Weber's ratios $\Delta i / i$, which are the functions of background intensity $i$, are illustrated for $\Delta v /\left(v_{\mathrm{m}}-v_{0}\right)=0 \cdot 1$. The curves in the case of power function are shown in Fig. 4 by using equations (11), (12) and (28). The

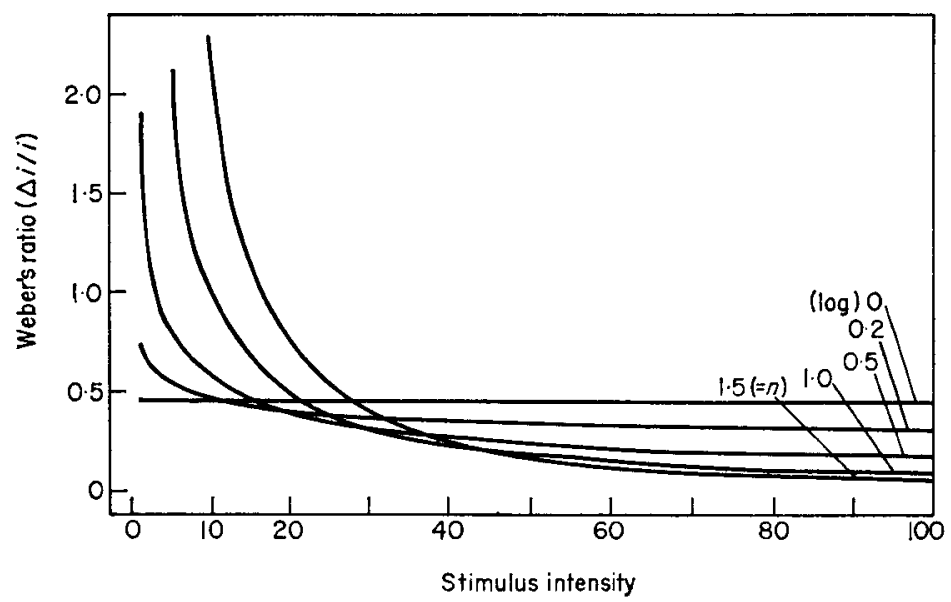

FIG. 4. The Weber's ratios $(\Delta i / i)$ in the receptor with power transformations and constant noise. The axes of co-ordinates are uniform scales. For $n=1.5,1.0,0.5$ and 0.2

For $n=0(\log )$

$$
\frac{\Delta i}{i}=\frac{1}{10} \frac{100^{n}-1}{n i^{n}}
$$

$$
\frac{\Delta i}{i}=\frac{1}{10} \log 100
$$

axes of co-ordinates are uniform scales. The curves in the case of tanh log function are shown in Fig. 5 by using equations (11), (14) and (28). The axes of co-ordinates are logarithmic scales. The curves of the latter have an axis of symmetry.

The relation between the probability distribution functions of the stimulus intensity and the potential amplitude is obtained by substituting $k=1$ into equation (42).

$$
\frac{v-v_{0}}{v_{\mathrm{m}}-v_{0}}=\frac{g(i)-g\left(i_{0}\right)}{g\left(i_{\mathrm{m}}\right)-g\left(i_{0}\right)}=\frac{h(i)-h\left(i_{0}\right)}{h\left(i_{\mathrm{m}}\right)-h\left(i_{0}\right)} .
$$

Although equation (43) has the same form as equation (9), they never indicate the same meaning. The differential of the right side of equation (43) 


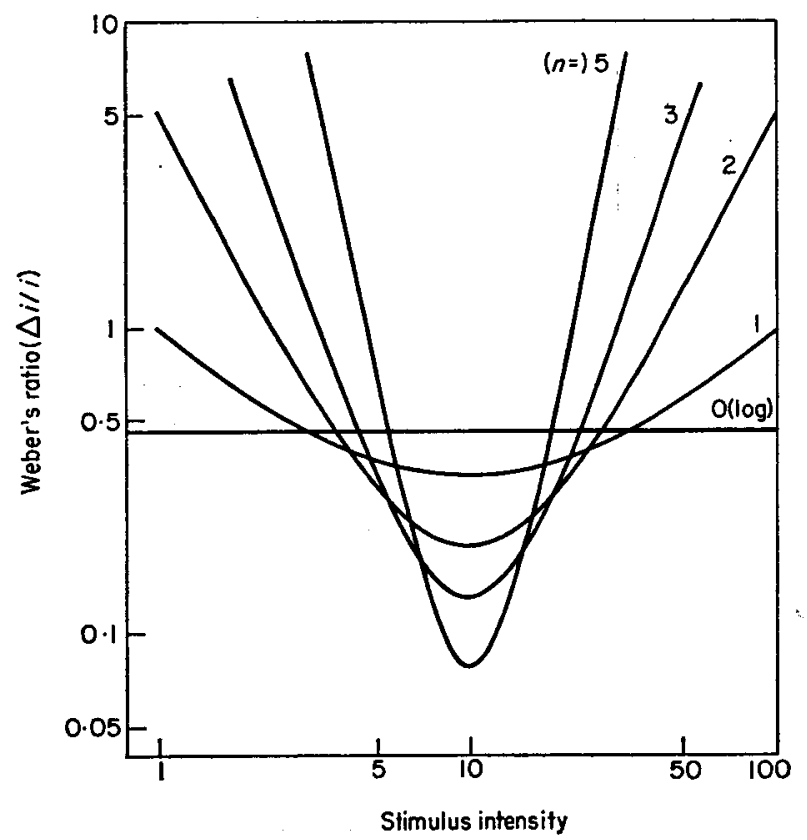

FIG. 5. The Weber's ratios $(\Delta i / i)$ in the receptor with tanh log transformations and constant noise. The axes of co-ordinates are logarithmic scales. For $n=5,3,2$ and 1

For $n=0(\log )$

$$
\frac{\Delta i}{i}=\frac{1}{10} \frac{\tanh \left(\frac{n}{4} \log 100\right)}{1-\left\{\tanh \left(\frac{n}{2} \log \frac{i}{10}\right)\right\}^{2}} \frac{4}{n}
$$

$$
\frac{\Delta i}{i}=\frac{1}{10} \log 100 \text {. }
$$

that is the distribution function of the stimulus intensity, produces the optimum p.d.f. of the stimulus intensity for the sensory nerve possessing a given stimulus-response relation. Namely,

$$
q(i)=\frac{h^{\prime}(i)}{h\left(i_{\mathrm{m}}\right)-h\left(i_{0}\right)} .
$$

The shapes of probability density distributions expressed by equation (44) are shown in Fig. 6 in the case of power function by using equations (1) and (2), then in Fig. 7 in the case of tanh $\log$ function by using equations (1) and (3). The abscissa of the stimulus intensity is a uniform scale and the ordinate of the probability density is a logarithmic scale. 


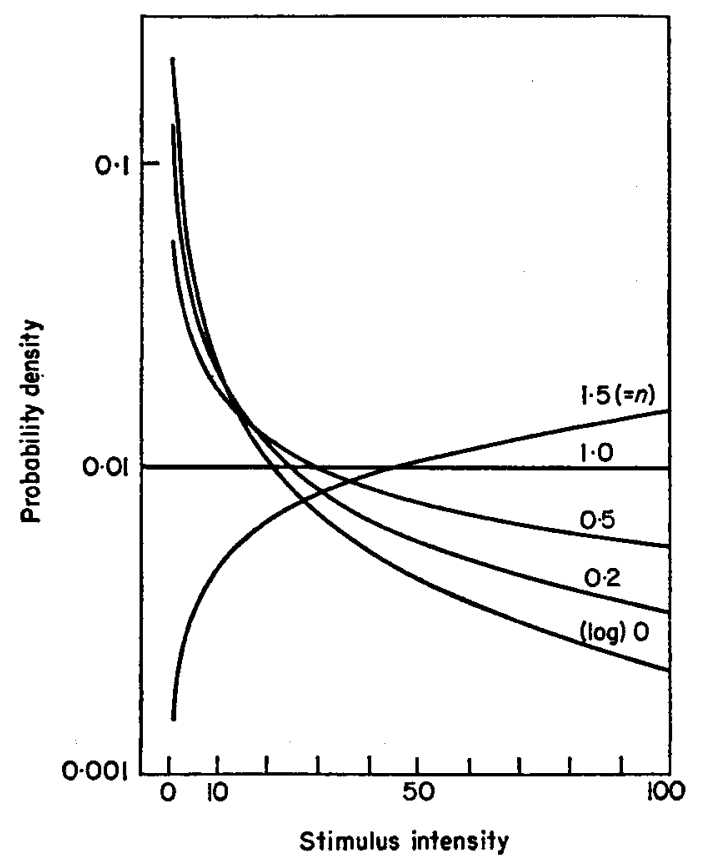

FIG. 6. The optimum probability density distributions of the stimulus intensity in the receptor with power transformations and constant noise. For $n=1.5,1.0,0.5$ and 0.2

For $n=0 \log$

$$
q(i)=\frac{n}{100^{n}-1} i^{n-1} .
$$

$$
q(i)=\frac{1}{\log 100} i^{-1}
$$

Werner \& Mountcastle (1965) studied stimulus-response relations for mechanoreceptive fibres ending in Iggo corpuscles of the skin of cats and monkeys. They expressed the stimulus intensities $(S)$ for each fibre as the per cent of maximum stimulus intensity used for that fibre $\left(S_{\max }\right)$; then the response magnitudes $(R)$ as per cents of the maximum response to $S_{\max }$ for each individual fibre. It was proved that the new, normalized variables were related in this form,

$$
R=(100)^{(1-n)} S^{n} .
$$

Here, the proportionality coefficients are represented by a function of the exponent $n$. Equation (12) can be rewritten as

$$
v=(100)^{(1-n)} i^{n}
$$




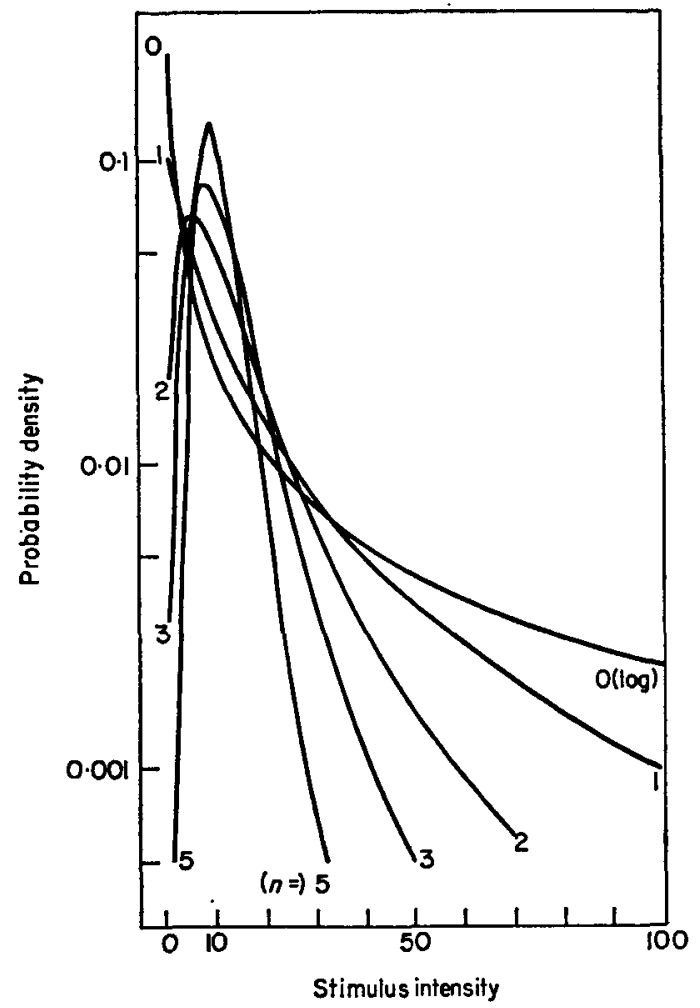

FrG. 7. The optimum probability density distributions of the stimulus intensity in the receptor with tanh $\log$ transformations and constant noise. For $n=5,3,2$ and 1

For $n=0(\log )$

$$
q(i)=\frac{10^{n}+1}{10^{n}-1} \frac{10^{n} n i^{n-1}}{\left(i^{n}+10^{n}\right)^{2}} .
$$

$$
q(i)=\frac{1}{\log 100} i^{-1}
$$

when we substitute $0(\%)$ for $i_{0}$ and $v_{0}$, and $100(\%)$ for $i_{\mathrm{m}}$ and $v_{\mathrm{m}}$. Formula (46) coincides with the empirical formula (45). Since $i_{0}$ is equal to zero, in the limit as the exponent $n$ approaches zero, equation (46) cannot change into the logarithmic formula.

I wish to express my thanks to Prof. I. Honjo for the discussion of the problems and the members of Photobiology Group for advice and encouragement. Thanks are also due to $\mathrm{Dr} \mathrm{H}$. Tamura for valuable suggestions and $\mathrm{Dr} \mathrm{K}$. Sugata for discussion. 


\section{REFERENCES}

BAYlor, D. A. \& Fuortes, M. G. F. (1970). J. Physiol. 207, 77.

BeIDLER, L. M. (1954). J. gen. Physiol. 38, 133.

BOYNTON, R. M. \& WHITTEN, D. V. (1970). Science, N.Y. 170, 1423.

Cox, D. R. \& MIILER, H. D. (1965). The Theory of Stochastic Processes. London: Methuen \& Co. Ltd.

ERNST, W. \& KeMP, C. M. (1972). Vision Res. 12, 1937.

Eyzaguirre, C. \& Kuffler, S. W. (1955). J. gen. Physiol. $39,87$.

Fano, R. M. (1963). Transmission of Information, A Statistical Theory of Communications. Cambridge: The M.I.T. Press.

FuORTES, M. G. F. (1958). Am.J. Ophthal. 46, 210.

Fuortes, M. G. F. (1970). Handbook of Sensory Physiology, vol. 1, Principle of Receptor Physiology, p. 243. Berlin, Heidelberg and New York: Springer-Verlag.

Galambos, R. \& Davis, H. (1943). J. Neurophysiol. 6, 39.

Hartline, H. K. \& Graham, C. H. (1932). J. cell. comp. Physiol. 1, 277.

KATZ, B. (1950). J. Physiol. 111, 261.

Matthews, B. H. C. (1931). J. Physiol. 71, 64.

NAKA, K. I. \& KishIDA, K. (1966). The Functional Organization of the Compound Eye, p. 251. Oxford: Pergamon Press.

STEIN, R. B. (1967). Biophys. J. 7, 797.

SteVENs, S. S. (1961). Sensory Communication, vol. 1. Cambridge: The M.I.T. Press.

Werner, G. \& MountCastle, V. B. (1965). J. Neurophysiol. 28, 359. 